\title{
INNER PRODUCT SPACES ASSOCIATED WITH POINCARÉ COMPLEXES
}

BY

\author{
SEIYA SASAO AND HIDEO TAKAHASHI
}

\begin{abstract}
We consider the homotopy type classification of a certain kind of Poincaré complex. First we define an inner product space associated with such a Poincare complex and we investigate the relation between the inner product space and the homotopy type of the Poincare complex. As an application, some results for manifolds are proved.
\end{abstract}

0. Introduction. Let $M$ be an oriented closed manifold of dimension $2 n$. It is well known that the bilinear map

$$
H^{n}(M ; Z) \times H^{n}(M ; Z) \rightarrow Z, \quad\left((x, y) \rightarrow\left\langle x \cup y, \mu_{M}\right\rangle\right)
$$

makes $H^{n}(M ; Z)$ an inner product space (in the sense of [2]) if $M$ has no torsion. This inner product space is closely related with the homotopy type of $M$. For example, J. Milnor has proved in [2, p. 103] the following (cohomology version).

TheOREM. Let $M_{i}(i=1,2$,$) be simply connected closed manifolds of dimension 4$. Their inner product spaces are isomorphic to each other if and only if they have the same oriented homotopy type.

We are interested in a generalization of Milnor's theorem to the case of torsion. In $\$ 1$ we shall define an inner product space over $Q / Z$ associated with an oriented Poincare complex and in $\$ \S 2-4$ apply this to the homotopy type classification of Poincare complexes $K$ which satisfy the conditions

(1) $K$ is $(n-1)$-connected and of dimension $2 n+1$,

(2) $H_{n}(K ; Z)$ is a finite abelian group $G$ without 2-torsion $(n \geqslant 3)$.

We call $K$ such as above a Poincaré complex of type $P^{n}(G)$ and in $\$ 2$ we shall discuss a special case $G=\Sigma^{s} Z_{p^{i}}$. In $\$ 3$ we shall give a decomposition theorem.

Theorem A. Let $K$ be a Poincaré complex of type $P^{n}(G)$ and let $G=\Sigma G_{p}^{i}$ be a direct sum decomposition of $G$, where $G_{p}^{i}$ is the $p^{i}$-component of $G$. Then $K$ has the same oriented homotopy type as the connected sum of Poincaré complexes of type $P^{n}\left(G_{p}^{i}\right)(i=1,2, \ldots$, odd primes $p)$.

Finally, in $\$ 4$ we prove

Theorem B. Let $K$ and $K^{\prime}$ be $S$-reducible Poincaré complexes of type $P^{n}(G)$. Then $K$ has the same oriented homotopy type as $K^{\prime}$ if and only if their product spaces are isomorphic.

Received by the editors December 12, 1978 and, in revised form, April 5, 1979 and October 19, 1979. AMS (MOS) subject classifications (1970). Primary 55D15. 
As an application we also prove

TheOREM C. Let $M$ be a closed smooth manifold whose underlying Poincaré complex is of type $P^{n}(G)$. Then if $n \neq 3 \bmod 4$ the oriented homotopy type of $M$ is determined by the isomorphism classes of the inner product space associated with $M$.

REMARK. In the case $n \equiv 3 \bmod 4$, Theorem $C$ also holds for $M$ with trivial Pontrjagin class $P_{n+1 / 4}(M)$.

In particular, for a manifold which is of type $P^{n}(G)$ we have

Corollary C-1. If $n \equiv 0 \bmod 2$, the oriented homotopy type of $M$ is uniquely determined by $H_{n}(M ; Z)$.

Corollary C-2. If $n \equiv 1 \bmod 4, M$ is the connected sum \# $M_{p^{i}, k}$ up to oriented homotopy equivalence, where $M_{p^{i}, k}$ is a closed manifold whose underlying Poincaré complex is of type $P^{n}\left(Z_{p^{i}}\right)$.

In $\S \S 1-4$ we always assume that $n \geqslant 3$ and $p$ is an odd prime number.

1. Inner product spaces. Let $K$ be a simply connected Poincare complex with the fundamental class $\mu_{K} \in H_{2 n+1}(K ; Z)$. Let $\delta: H^{*}(K ; Q / Z) \rightarrow H^{*+1}(K ; Z)$ be the connecting homomorphism associated with the exact sequence of coefficients, $0 \rightarrow Z \rightarrow Q \rightarrow Q / Z \rightarrow 0$.

We consider the bilinear map

$$
\beta_{K}: H^{n}(K ; Q / Z) \times H^{n}(K ; Q / Z) \rightarrow Q / Z
$$

defined by the composite map

$$
\begin{aligned}
H^{n}(K ; Q / Z) \times H^{n}(K ; Q / Z) & \stackrel{1 \times \delta}{\rightarrow} H^{n}(K ; Q / Z) \times H^{n+1}(K ; Z) \\
& \stackrel{1 \times D}{\rightarrow} H^{n}(K ; Q / Z) \times H_{n}(K ; Z) \stackrel{<,\rangle}{\rightarrow} Q / Z,
\end{aligned}
$$

where $D$ is the Poincare duality map and $\langle$,$\rangle denotes the Kronecker pairing. The$ following is well known,

LEMMA 1.1. $\beta_{K}(x, y)=(-1)^{n+1} \beta_{K}(y, x)$.

Moreover assuming that $H^{n}(K ; Z)$ (and hence $H^{n+1}(K ; Z)$ ) are torsion, we have

Proposition 1.2. $\beta_{K}$ is a completely orthogonal pairing (cf. [2]) and symmetric, for odd $n$, and skew symmetric, for even $n$.

Proof. Clearly the latter follows from Lemma 1.1. The former follows from the Poincaré duality theorem for torsion groups.

Thus we have the inner product space $V(K)=\left\{H^{n}(K ; Q / Z), \beta_{K}\right\}$ associated with a Poincaré complex $K$. Clearly this inner product space is an oriented homotopy type invariant for oriented Poincaré complexes and we are interested in the problem, "Is the converse true?" The next section investigates a special case.

2. A special case $G=\Sigma^{s} Z_{p^{i}}$. Let $M\left(n, p^{i}\right)$ be the Moore space of type $\left(n, Z_{p^{i}}\right)$. Note the following easy

$$
\text { LEMMA 2.1. } \pi_{n+1}\left(M\left(n, p^{i}\right)\right)=0=\pi_{n+2}\left(M\left(n, p^{i}\right)\right) \text {. }
$$


To study the homotopy type classification of Poincaré complexes which are $(n-1)$-connected and of dimension $2 n+1$ having $H_{n}(K ; Z)=\Sigma^{s} Z_{p^{i}}$, we must investigate the homotopy group $\pi_{2 n}\left(M_{s}\right)$ where $M_{s}$ denotes the wedge sum of $s$ copies of $M\left(n, p^{i}\right)$. Clearly a Poincaré complex $K$ has the same homotopy type as the mapping cone of a map $f: S^{2 n} \rightarrow M_{s}$. First we note the following two lemmas which are proved by standard arguments.

LEMMA 2.2. The smash product $M\left(n, p^{i}\right) \wedge M\left(n, p^{i}\right)$ is the wedge sum $M\left(2 n, p^{i}\right)$ $\bigvee M\left(2 n+1, p^{i}\right)$ up to homotopy.

LEMMA 2.3. $\pi_{2 n}\left(M_{s}\right)=\pi_{2 n}\left(M_{s-1}\right) \oplus \pi_{2 n}\left(M_{1}\right) \oplus \pi_{2 n+1}\left(M_{s-1} \wedge M_{1}\right)$.

Secondly we consider the special case $s=2$. Let $P$ be the natural map $M\left(n, p^{i}\right)$ $\rightarrow S^{n+1}=M\left(n, p^{i}\right) / S^{n}$ and consider the map

$$
\hat{P}=\mathrm{id} \vee P: M\left(n, p^{i}\right) \vee M\left(n, p^{i}\right) \rightarrow M\left(n, p^{i}\right) \vee S^{n+1}
$$

Then we have the commutative diagram,

$$
\begin{array}{ccccc}
\pi_{2 n}\left(M_{2}\right) & =\pi_{2 n}\left(M_{1}\right) \oplus & \pi_{2 n}\left(M_{1}\right) & \oplus & \pi_{2 n+1}\left(M_{1} \times M_{1}, M_{2}\right) \\
\downarrow \hat{P}_{*} & \searrow \mathrm{id} & \searrow P_{*} & \searrow(\mathrm{id} \times P)_{*} \\
\pi_{2 n}\left(M_{1} \vee S^{n+1}\right)=\pi_{2 n}\left(M_{1}\right) \oplus \pi_{2 n}\left(S^{n+1}\right) \oplus & \pi_{2 n+1}\left(M_{1} \times S^{n+1}, M_{1} \vee S^{n+1}\right),
\end{array}
$$

in which $(\mathrm{id} \times P)_{*}$ is isomorphic because we have isomorphisms

$$
\begin{array}{cccc}
\pi_{2 n+1}\left(M_{1} \times M_{1}, M_{1} \vee M_{1}\right) & \stackrel{\sim}{ } & H_{2 n+1}\left(M_{1} \times M_{1}, M_{1} \vee M_{1}\right) \\
\downarrow(\mathrm{id} \times P)_{*} & & \downarrow & \approx \\
\pi_{2 n+1}\left(M_{1} \times S^{n+1}, M_{1} \vee S^{n+1}\right) & \stackrel{\approx}{\rightarrow} & H_{2 n+1}\left(M_{1} \times S^{n+1}, M_{1} \vee S^{n+1}\right) .
\end{array}
$$

Let $\iota$ be the inclusion

$$
S^{n} \hookrightarrow M_{1}=M\left(n, p^{i}\right)=S^{n} \cup_{p^{i}} e^{n+1} .
$$

Since it is easily seen that the Whitehead product $\left[\imath, \iota_{n+1}\right]$ generates the group $\pi_{2 n+1}\left(M_{1} \times S^{n+1}, M_{1} \vee S^{n+1}\right)$, we can take the element $\alpha=\hat{P}_{*}^{-1}\left(\left[\iota, \iota_{n+1}\right]\right)$ as a generator for the group $\partial \pi_{2 n+1}\left(M_{1} \times M_{1}, M_{2}\right)$ using the above diagram. Let $i_{k}$ be the inclusion of $M_{1}$ into the $k$ th factor of $M_{s}$ and $i_{k, l}$ be the map $M_{2}=M_{1} \vee M_{1} \rightarrow$ $M_{s}\left(i_{k, l}=i_{k} \vee i_{l}\right)$. Then by Lemmas 2.2 and 2.3, we have

Proposition 2.4. $\pi_{2 n}\left(M_{s}\right)=\Sigma^{s} \pi_{2 n}\left(M_{1}\right) \oplus \Sigma Z_{p}\left[\alpha_{k, l}\right]$, where the second summation runs over $1 \leqslant k<l \leqslant s$ and $\alpha_{k, l}=i_{k, l *}(\alpha)$.

Let $x_{0}$ be the fixed generator of $H^{n}\left(M\left(n, p^{i}\right) ; Q / Z\right) \approx Z_{p^{i}}$ and let $p_{k}$ be the projection $M_{s} \rightarrow M_{1}$ onto the $k$ th factor of $M_{s}$. We take $\left\{x_{k}=p_{k}^{*}\left(x_{0}\right)\right\}$ as a system of generators for $H^{n}\left(M_{s} ; Q / Z\right)$ and identify $H^{n}\left(M_{s} ; Q / Z\right)$ with $H^{n}\left(K_{f} ; Q / Z\right)$ for the mapping cone $K_{f}$ of a map $f: S^{2 n} \rightarrow M_{s}$. Now we define a homomorphism

$$
D_{s}: \pi_{2 n}\left(M_{s}\right) \rightarrow\{s \times s \text {-matrices over } Q / Z\}
$$

by the formula

$$
D_{s}(f)=\left(f_{k, l}\right), \quad f_{k, l}=\left\langle x_{k}, \mu_{f} \cap \delta x_{l}\right\rangle,
$$

where $\mu_{f}$ denotes the oriented generator of $H_{2 n+1}\left(K_{f} ; Z\right)$. 
LEMMA 2.5. The matrix $\left(f_{k, l}\right)$ is symmetric for odd n and skew symmetric for even $n$. Moreover, $K_{f}$ is a Poincaré complex if and only if $D_{s}(f)=\left(f_{k, l}\right)$ is invertible.

Proof. The first part follows from Lemma 1.1. The second part is equivalent to Poincaré duality.

Let $\varphi$ be a map $M_{s} \rightarrow M_{t}$ and define the matrix $U=\left(u_{k, l}\right)\left(u_{k, l} \in Q / Z\right)$ by $\varphi^{*}\left(x_{k}\right)=\Sigma_{l} u_{k, l} x_{l}(k=1, \ldots, t)$. Then by the definition of $D_{s}$ and $U$, we have

LeMma 2.6 (NATURALITY OF $D_{s}$ ). For a map $\varphi: M_{s} \rightarrow M_{t}$, we have

$$
D_{t}\left(\varphi_{*}(f)\right)=U \circ D_{s}(f) \circ{ }^{t} U \quad\left(f \in \pi_{2 n}\left(M_{s}\right)\right) .
$$

Now we prove a key lemma for our purpose.

Lemma 2.7. Let $f=\sum f_{k}+\sum a_{k, l}\left[\alpha_{k, l}\right](k<l)$ be the decomposition in Proposition 2.4. Then we have

$$
f_{k, l}=a_{k, l} \quad(k<l) \text { and } f_{k, k}=D_{1}\left(f_{k}^{\prime}\right)
$$

where $D_{s}(f)=\left(f_{k, l}\right)$ and $i_{k^{*}}\left(f_{k}^{\prime}\right)=f_{k}$.

Proof. The equation $f_{k, k}=D_{1}\left(f_{k}^{\prime}\right)$ follows from the naturality for the projection $p_{k}: M_{s} \rightarrow M_{1}\left(p_{k}(f)=f_{k}^{\prime}\right)$. For $f_{k, l}=a_{k, l}$, it is sufficient to prove $D_{s}\left(\alpha_{k, l}\right)=\{(k, l)$ and $(l, k)$ components are \pm 1 and others are 0$\}$, and this is equivalent to $D_{2}(\alpha)=\left(\begin{array}{r}0 \\ \pm 1 \\ 1\end{array}\right)$ by the naturality. And so we consider as follows:

Now there exists a map $\lambda: K_{\alpha} \rightarrow M_{1} \times M_{1}$ such that $\lambda \mid M_{2}=\mathrm{id}_{M_{2}}: M_{2} \rightarrow M_{2} \subset$ $M_{1} \times M_{1}$ and $\lambda_{*}\left(\mu_{\alpha}\right)$ is a generator of $H_{2 n+1}\left(M_{1} \times M_{1} ; Z\right) \approx Z_{p i}$. Then we consider the following diagram:

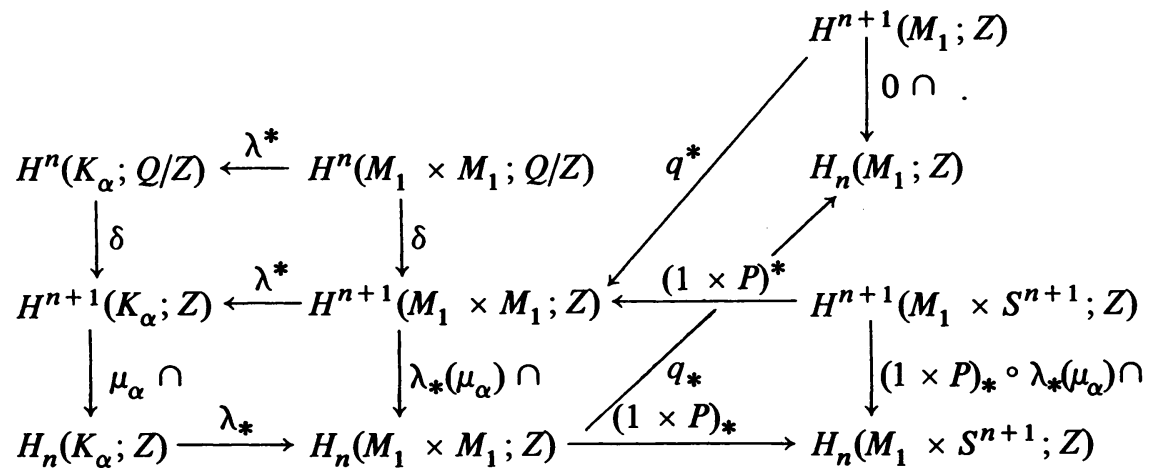

where $P$ denotes the natural collapsing map $M_{1} \rightarrow M_{1} / S_{n}$ and $q$ denotes the projection $M_{1} \times M_{1} \rightarrow M_{1}$ onto the first factor. Then it is easily verified that $D_{2}(\alpha)=\left(\begin{array}{c}0 \\ \pm 1 \\ 0\end{array}\right)$ using the standard generators of $H_{n}\left(K_{\alpha} ; Z\right)$ and $H^{n+1}\left(K_{\alpha} ; Z\right)$. Thus the proof of the lemma is completed.

Here we recall that for two matrices $A$ and $B$ over a ring $R A \equiv B$ if and only if there exists an invertible matrix $U$ such that $U \circ A \circ{ }^{t} U=B$. Then the preceding argument shows

LEMMA 2.8. $D_{s}(f) \equiv D_{s}(g)$ for $f, g \in \pi_{2 n}\left(M_{s}\right)$ if and only if there exists a homotopy equivalence $\varphi: M_{s} \rightarrow M_{s}$ such that $\varphi_{*}(f) \equiv g \bmod D_{s}^{-1}(0)$. 
Let $K_{i}(i=1,2)^{\cdot}$ be two Poincaré complexes of type $P^{n}\left(\Sigma^{s} Z_{p^{i}}\right)$ and we shall prove the main proposition in this section.

Proposition 2.9. If $K_{i}$ are both $S$-reducible, then $K_{1}$ has the same homotopy type as $K_{2}$ if and only if $V\left(K_{1}\right)$ is isomorphic to $V\left(K_{2}\right)$.

Let

$$
E^{N}: \pi_{2 n}\left(M_{s}\right) \rightarrow \pi_{2 n+N}\left(E^{N} M_{s}\right) \quad(N \rightarrow \infty)
$$

denote the suspension homomorphism. Specially we use the restricted homomorphism

$$
E^{N}: D_{s}^{-1}(0) \rightarrow \pi_{2 n+N}\left(E^{N} M_{s}\right) .
$$

Since $D_{s}^{-1}(0)$ is decomposed into the direct sum $\sum i_{k^{*}} D_{1}^{-1}(0)$ by Proposition 2.4, it is sufficient for us to consider the case $s=1$. Then from Diagram 1.6 of [4] $\left(h_{n}\right.$ is essentially $D_{1}$ ), we get

LEMMA 2.10. $E^{N}: D_{1}^{-1}(0) \rightarrow \pi_{2 n+N}\left(E^{N} M_{1}\right)$ is injective.

Now we prove Proposition 2.9. Since $K_{1}$ has the same homotopy type as the mapping cone $K_{f_{1}}$ for a map $f_{1}: S^{2 n} \rightarrow M_{s}$, the inner product space $V\left(K_{1}\right)$ is isomorphic to $V\left(K_{f_{1}}\right)$. If $V\left(K_{f_{1}}\right)$ is isomorphic to $V\left(K_{f_{2}}\right)$ for another map $f_{2}$ : $S^{2 n} \rightarrow M_{s}$, there exists a homotopy equivalence $\varphi: M_{s} \rightarrow M_{s}$ such that $\varphi_{*}\left(f_{1}\right) \equiv f_{2}$ $\bmod D_{s}^{-1}(0)$, by Lemma 2.8. Since the $S$-reducibility of $K_{i}$ means $E^{N}\left(f_{i}\right)=0$ $(N \rightarrow \infty)$, the proof is completed from Lemma 2.10 .

Corollary 2.11. Let $K$ be a Poincaré complex of type $P^{n}\left(\Sigma^{s} Z_{p^{i}}\right)$. Then if $n$ is odd, $K$ is the connected sum of Poincaré complexes of type $P^{n}\left(Z_{p^{i}}\right)$ up to homotopy.

Corollary 2.12. If $n$ is even, the homotopy type for $S$-reducible Poincaré complex of type $P^{n}\left(\Sigma^{s} Z_{p^{i}}\right)$ is uniquely determined by $s$.

Proof. $K$ is the mapping cone $K_{f}$ up to homotopy and we may assume that $D_{s}(f)$ is a diagonal matrix if $n$ is odd. Hence $f$ has the decomposition $f=\Sigma f_{k}$ in Proposition 2.4. This means that $K_{f}$ is the connected sum $\#^{s} K_{f_{k}}$ up to homotopy. Since $K_{f_{k}}$ is a Poincaré complex of type $P^{n}\left(Z_{p^{i}}\right)$, the proof of Corollary 2.11 is completed. Moreover since we know that $D_{s}(f)$ is unique up to equivalence if $n$ is even, Corollary 2.12 follows from Proposition 2.9.

3. Decomposition (up to homotopy). First we define a generalized Hopf homomorphism $H_{G}: \pi_{2 n}(M(n, G)) \rightarrow$ End $G$ by the formula

$$
H_{G}(f)=\mu_{f} \cap: H^{n+1}\left(K_{f} ; Z\right)=G \rightarrow H_{n}\left(K_{f} ; Z\right)=G
$$

where $\mu_{f} \in H_{2 n+1}\left(K_{f} ; Z\right)$ denotes the oriented generator of the mapping cone $K_{f}$ for a map $f: S^{2 n} \rightarrow M(n, G)$. Let $\rho: G \rightarrow H$ be a homomorphism and $\hat{\rho}: M(n, G)$ $\rightarrow M(n, H)$ be the map induced by $\rho\left(\rho=\hat{\rho}_{*}: H_{n}(M(n, G) ; Z)=G \rightarrow\right.$ $\left.H_{n}(M(n, H) ; Z)=H\right)$. If we denote by ' $\rho$ the homomorphism

$$
\rho^{*}: H^{n+1}(M(n, H) ; Z)=H \rightarrow H^{n+1}(M(n, G) ; Z)=G,
$$

then we have 
LeMMA 3.1. $H_{H}(\hat{\rho} \circ f)=\rho \circ H_{G}(f) \circ{ }^{t} \rho$ for $f \in \pi_{2 n}(M(n, G))$.

Proof. Let $\hat{\rho}_{f}: K_{f} \rightarrow K_{\hat{\rho}}$ 。f be the natural extension of $\hat{\rho}$ and consider the diagram

$$
\begin{array}{ccc}
H^{n+1}\left(K_{f} ; Z\right)=G & \overrightarrow{\mu_{f} \cap} & H_{n}\left(K_{f} ; Z\right)=G \\
\uparrow \rho_{f}^{*} & \downarrow \rho_{f_{\bullet}} \\
H^{n+1}\left(K_{\hat{\rho} \circ f} ; Z\right)=H & \underset{\mu \hat{\rho}_{\hat{\rho} \circ}}{\rightarrow} \cap & H_{n}\left(K_{\hat{\rho} \circ f} ; Z\right)=H
\end{array}
$$

Then the proof follows from the commutativity of the diagram.

Let $G=G_{1} \oplus G_{2}$ be a direct sum decomposition of $G$. From Lemma 3.1 we have the diagram

$$
\begin{aligned}
& \pi_{2 n}(M(n, G))=\pi_{2 n}\left(M\left(n, G_{1}\right)\right) \oplus \pi_{2 n}\left(M\left(n, G_{2}\right)\right) \oplus \pi_{2 n+1}\left(M\left(n, G_{1}\right) \times M\left(n, G_{2}\right), \bigvee\right) \\
& \downarrow H_{G} \quad \downarrow H_{G_{1}} \quad \downarrow H_{G_{2}} \quad \leadsto \\
& \text { End } G=\text { End } G_{1} \quad \oplus \quad \text { End } G_{2} \quad \oplus \operatorname{Hom}\left(G_{1}, G_{2}\right) \oplus \operatorname{Hom}\left(G_{2}, G_{1}\right) \text {. }
\end{aligned}
$$

LEMMA 3.3. The restriction $H_{G} \mid \partial \pi_{2 n+1}\left(M\left(n, G_{1}\right) \times M\left(n, G_{2}\right), \vee\right)$ is injective.

Proof. Let $G_{1}=\sum Z_{p^{i}}\left[x_{p, i, l}\right]$ and $G_{2}=\sum Z_{p}\left[y_{p, j, k}\right]$ be a direct sum decomposition of $G_{1}$ and $G_{2}$ respectively. Since we have decompositions

$$
\begin{aligned}
\pi_{2 n+1}\left(M\left(n, G_{1}\right) \times M\left(n, G_{2}\right), M\left(n, G_{1}\right) \vee M\left(n, G_{2}\right)\right) \\
=\pi_{2 n+1}\left(M\left(n, Z_{p^{i}}\right) \times M\left(n, Z_{p^{j}}\right), M\left(n, Z_{p^{i}}\right) \vee M\left(n, Z_{p^{j}}\right)\right)
\end{aligned}
$$

and $\operatorname{Hom}\left(G_{1}, G_{2}\right) \oplus \operatorname{Hom}\left(G_{2}, G_{1}\right)=\sum \operatorname{Hom}\left(Z_{p^{i}}, Z_{p^{j}}\right) \oplus \operatorname{Hom}\left(Z_{p^{j}}, Z_{p^{i}}\right)$, it is sufficient for us to consider the case $G_{1}=Z_{p^{i}}[x]$ and $G_{2}=Z_{p}[y]$. Thus the proof follows by an argument similar to the proof of Lemma 2.7 .

REMARK. We note that the proof also shows $H_{G}\left(f_{3}\right) \mid G_{2}={ }^{t}\left(H_{G}\left(f_{3}\right) \mid G_{1}\right)$ for $f=f_{1}+f_{2}+f_{3}$ in diagram (3.2).

LEMMA 3.4. Let $f=f_{1}+f_{2}+f_{3}$ be the decomposition of $f$ in diagram (3.2). If $H_{G_{1}}\left(f_{1}\right)$ is contained in Aut $G_{1}$, then there exists a homotopy equivalence $h: M(n, G)$ $\rightarrow M(n, G)$ such that $h_{*}(f)$ is contained in $\pi_{2 n}\left(G_{1}\right) \oplus \pi_{2 n}\left(G_{2}\right)$ and $H_{G_{1}}\left(f_{1}\right)=H_{G_{1}}\left(f_{1}^{\prime}\right)$ where $h_{*}(f)=f_{1}^{\prime}+f_{2}^{\prime}$.

Proof. If we show that there exists an isomorphism $\lambda: G \rightarrow G$ such that $\lambda \circ H_{G}(f) \circ{ }^{t} \lambda \mid$ End $G_{1}=0$ and $\lambda \circ H_{G}(f) \circ{ }^{t} \lambda \mid \operatorname{Hom}\left(G_{1}, G_{2}\right)=H_{G_{1}}\left(f_{1}\right)$, then the proof follows from Lemmas 3.1, 3.3 and the above remark.

For homomorphisms $\lambda_{i, j}: G_{i} \rightarrow G_{j}(i, j=1,2)$, we define a homomorphism $\lambda$ : $G \rightarrow G$ by $\lambda(g)=\lambda_{1,1}\left(g_{1}\right)+\lambda_{2,1}\left(g_{2}\right)+\lambda_{1,2}\left(g_{1}\right)+\lambda_{2,2}\left(g_{2}\right)$ for $g=g_{1}+g_{2}$. Then from easy computation, we can get

$$
\begin{aligned}
\lambda \circ h_{G}(f) \circ{ }^{t} \lambda \mid \text { End } G_{1}= & \lambda_{1,1} \circ H_{G_{1}}\left(f_{1}\right) \circ{ }^{t} \lambda_{1,1}+\lambda_{2,1} \circ H_{G}\left(f_{3}\right) \mid G_{1} \circ{ }^{t} \lambda_{1,1} \\
& +\lambda_{1,1} \circ H_{G}\left(f_{3}\right) \mid G_{2} \circ{ }^{t} \lambda_{2,1}+\lambda_{2,1} \circ H_{G_{2}}\left(f_{2}\right) \circ{ }^{t} \lambda_{2,1}
\end{aligned}
$$


and

$$
\begin{aligned}
\lambda \circ H_{G}(f) \circ{ }^{t} \lambda \mid \operatorname{Hom}\left(G_{1}, G_{2}\right)= & \lambda_{1,2} \circ H_{G_{L}}\left(f_{1}\right) \circ{ }^{t} \lambda_{1,1}+\lambda_{2,2} \circ H_{G}\left(f_{3}\right) \mid G_{1} \circ{ }^{t} \lambda_{1,1} \\
& +\lambda_{1,2} \circ H_{G}\left(f_{3}\right) \mid G_{2} \circ{ }^{t} \lambda_{2,1}+\lambda_{2,2} \circ H_{G_{2}}\left(f_{2}\right) \circ{ }^{t} \lambda_{2,1} .
\end{aligned}
$$

Hence if we take $\lambda_{1,1}=\mathrm{id}_{G_{1}}, \lambda_{2,2}=\mathrm{id}_{G_{2}}, \lambda_{2,1}=0$ and

$$
\lambda_{1,2}=-H_{G}\left(f_{3}\right) \mid G_{1} \circ H_{G_{1}}\left(f_{1}\right)^{-1} \text {, }
$$

then $\lambda$ satisfies our conditions.

Lemma 3.5. Suppose $G=G_{1} \oplus G_{2}$ where $G_{1}=\sum Z_{p^{m}}$ and $p^{m-1} G_{2}$ has a trivial p-primary component. If $H_{G}(f)$ is an automorphism for $f=f_{1}+f_{2}+f_{3}$, then $H_{G_{1}}\left(f_{1}\right)$ is also an automorphism.

Proof. Consider the following exact ladder

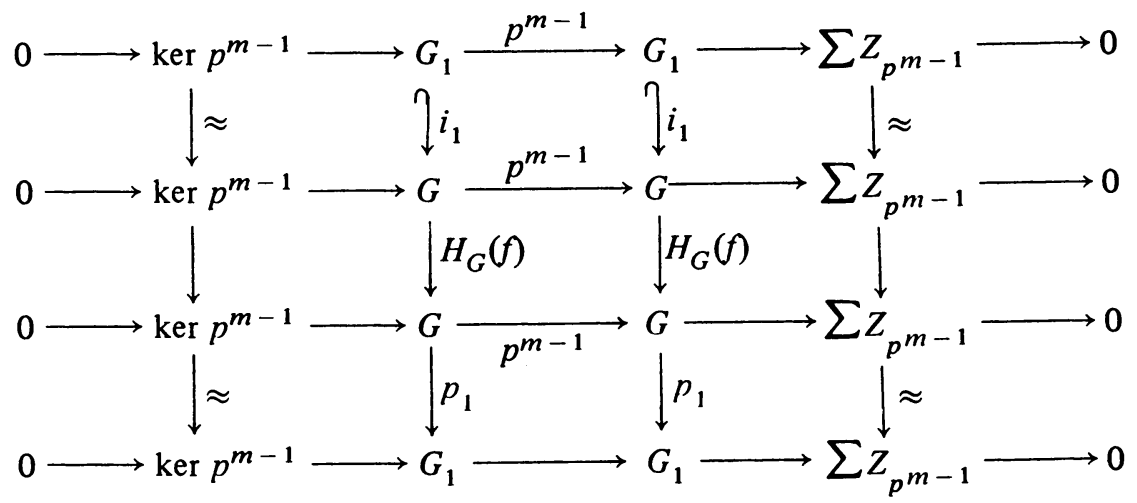

where $i_{1}$ is the inclusion $G_{1} \hookrightarrow G$ and $p_{1}$ is the projection $G \rightarrow G_{1}$ and $p^{m-1}$ denotes the map multiplied by $p^{m-1}$. The right and left vertical maps are isomorphisms because $H_{G}(f)$ is also. Hence it follows that $H_{G_{1}}\left(f_{1}\right)=p_{1} \circ H_{G}(f) \circ i_{1}$ is surjective and so an isomorphism. Now Theorem $A$ follows from Lemmas 3.4 and 3.5.

\section{Proofs of Theorems $B$ and $C$.}

Lemma 4.1. Let $K_{i}(i=1,2)$ be two Poincaré complexes of dimension $m$. Then the connected sum $K_{1} \# K_{2}$ is $S$-reducible if and only if $K_{1}$ and $K_{2}$ are both $S$-reducible.

Proof. This is clear because $K_{1} \# K_{2}$ has a CW-decomposition $\left(K_{1}^{(m-1)} \mathrm{V}\right.$ $\left.K_{2}^{(m-1)}\right) \cup_{f} e^{m}$, where $f=f_{1}+f_{2}$ for $K_{i}=K_{i}^{(m-1)} \cup_{f_{i}} e^{m}$.

Now we prove Theorem B. Let $K$ and $K^{\prime}$ be two Poincaré complexes of type $P^{n}(G)$. Trivially $V(K) \equiv V\left(K^{\prime}\right)$ if $K$ and $K^{\prime}$ have the same oriented homotopy type. Assume that $V(K) \equiv V\left(K^{\prime}\right)$. By Theorem A, we may assume that $K$ and $K^{\prime}$ are the connected sums \# $K_{p, i}$ and \# $K_{p, i}^{\prime}$ (corresponding to the $Z_{p^{i}}$-component of $G)$ respectively. Let $h_{i}: K \rightarrow K_{p, i}$ and $h_{i}^{\prime}: K^{\prime} \rightarrow K_{p, i}^{\prime}$ be the projection onto each factor. Since we may consider $\beta_{K}=\sum \beta_{K} \mid \sum Z_{p^{i}}$ and $\beta_{K^{\prime}}=\sum \beta_{K^{\prime}} \mid \sum Z_{p^{i}}$ by Lemmas 3.4 and $3.5, h_{i}\left(h_{i}^{\prime}\right)$ induces an isomorphism $V(K) \mid \sum Z_{p^{i}} \cong V\left(K_{p, i}\right)\left(V\left(K^{\prime}\right) \mid \sum Z_{p^{i}} \cong\right.$ $\left.V\left(K_{p, i}^{\prime}\right)\right)$ and so $V\left(K_{p, i}\right)$ is isomorphic to $V\left(K_{p, i}^{\prime}\right)$. Thus $K_{p, i}$ has the same oriented 
homotopy type as $K_{p, i}^{\prime}$ by Proposition 2.9 and Lemma 4.1, and hence $K$ has the same oriented homotopy type as $K^{\prime}$. Thus the proof is completed.

From Theorem A and Corollaries 2.11 and 2.12, we have

Corollary B-1. Let $K$ be a Poincare complex of type $P^{n}(G)$. Then $K$ has the same homotopy type as the connected sum of Poincaré complexes of type $P^{n}\left(Z_{p^{i}}\right)$ for various $p$ and $i$ if $n$ is odd.

COROllary B-2. If $\boldsymbol{n}$ is even, the oriented homotopy type for $S$-reducible Poincaré complexes of type $P^{n}(G)$ is uniquely determined by $n$ and $G$.

Let $M$ be a closed smooth manifold of dimension $2 n+1$ whose underlying Poincaré complex is of type $P^{n}(G)$ and let $\nu_{M}: M \rightarrow B O$ be the stable normal bundle for $M$. Since $M$ has the same homotopy type as the mapping cone $K_{f}$ for a map $f: S^{2 n} \rightarrow M(n, G)$, we may replace $M$ with $K_{f}$.

LEMMA 4.2. If $n \neq 3 \bmod 4$ then $\nu_{M}: K_{f} \rightarrow B O$ is trivial.

Proof. First consider the restriction $\nu_{M} \mid M_{1}$,

$$
K_{f} \supset M(n, G) \supset M_{1}=S^{n} \underset{p^{i}}{\cup} e^{n+1} \rightarrow B O .
$$

From Puppe's sequence and $\pi_{n}(B O)=0, Z$ or $Z_{2}$, we obtain that $\nu_{M} \mid M_{1}$ is trivial, and so $\nu_{M} \mid M(n, G)$ is trivial. Hence $\nu_{M}$ is decomposed as follows

$$
\nu_{M}: K_{f} \stackrel{P}{\rightarrow} S^{2 n+1} \stackrel{\nu}{\rightarrow} B O
$$

where $P$ denotes the natural map $K_{f} \rightarrow K_{f} / M(n, G)=S^{2 n+1}$. Since $P$ induces the map $T_{P}: T\left(\nu_{M}\right) \rightarrow T(\nu)$ of degree $1, T(\nu)$ is reducible and $\nu$ is fiber homotopically trivial, that is, $J(\nu)=0$ in this case. Hence $\nu$ is trivial by Adams' Theorem. This completes the proof.

Thus since Lemma 4.2 shows that $M$ is $S$-reducible, Theorem $\mathrm{C}$ and Corollary C-1 follow from Theorem B and Corollary B-2 respectively. Corollary C-2 is also obtained from Lemmas 4.1 and 4.2 and Corollary B-1.

For the case $n \equiv 3 \bmod 4$, the situation is more complicated and we could not obtain simple theorems. As an example we list

Proposition 4.3. Suppose (order $G$, order $\left.J\left(S^{n+1}\right)\right)=1$. For any element $x$ of $H^{n+1}(M(n, G) ; Z) \approx G$, there exists a closed smooth manifold $M^{2 n+1}$ with $P_{n+1 / 4}(M)=x$ which is of type $P^{n}(G)$ as a Poincaré complex and is a $\pi$-manifold up to homotopy.

Proof. The proof follows from the case $G=Z_{p}$, using the connected sum operation. On the other hand, the condition $\left(p\right.$, order $\left.J\left(S^{n+1}\right)\right)=1$ means that $J\left(K_{f}\right)=0$ for any $f \in \pi_{2 n}\left(M\left(n, Z_{p^{i}}\right)\right)$. Since we can take $f$ such that $D_{1}(f)=1$ and $E^{N}(f)=0$, the proof is completed. 


\section{REFERENCES}

1. W. Browder, Surgery on simply-connected manifolds, Ergebnisse der Mathematik und ihrer Grenzgebiete, Band 65, Springer-Verlag, New York-Heidelberg, 1972.

2. J. Milnor and D. Husemoller, Symmetric bilinear forms, Ergebnisse der Mathematik und ihrer Grenzgebiete, Band 73, Springer-Verlag, New York and Berlin.

3. C. T. C. Wall, Periodicity in algebraic L-theory, Manifolds-Tokyo 1973, Univ. Tokyo Press, Tokyo, 1975, pp. 57-68.

4. S. Sasao and H. Takahashi, Highly connected Poincaré complexes, Kodai Math. J. 2 (1979), 139-147.

Department of Mathematics, Tokyo Institute of Technology, Oh-OKayama, Meguro-ku, TOKYO, JAPAN (Current address of Seiya Sasao)

Current address (Hideo Takahashi): Department of Mathematics, Technological University of Nagaoka, Kamitomioka-cho, Nagaoka, Japan 